\title{
Measures and LMIs for Validation of an Aircraft with MRAC and Uncertain Actuator Dynamics
}

\author{
Daniel Wagner* \\ Faculty of Electrical Engineering, Czech Technical University in Prague, 16626, Czech Republic \\ Didier Henrion ${ }^{\dagger}$ \\ Faculty of Electrical Engineering, Czech Technical University in Prague, 16626, Czech Republic \\ LAAS, CNRS, Toulouse, 31400, France \\ Martin Hromčík \\ Faculty of Electrical Engineering, Czech Technical University in Prague, 16626, Czech Republic
}

It is well known that actuator dynamics with degraded performance can lead to poor stability and tracking for adaptive control. In this paper, we use occupation measures and LMI relaxations (called the moment sums of squares or Lasserre hierarchy) for verification and validation of a longitudinal F-16 aircraft model with MRAC and higher order actuator dynamics with uncertain parameters and deflection saturation. These uncertain parameters are represented explicitly in the space of occupation measures. To improve numerical scalability, we exploit sparsity for ordinary differential equations (ODEs) using parsimony to partition the dynamics. We finally compare these main results to those obtained using Monte-Carlo methods.

\section{Nomenclature}

$\alpha(t) \quad=$ angle of attack [rad]

$q(t)=$ pitchrate $[\mathrm{rad} / \mathrm{s}]$

$v(t)=$ elevator actuator output [deg]

$\mathbf{x}_{\mathbf{p}}(t)=$ short-period longitudinal dynamics

$\mathbf{x}_{\mathbf{c}}(t)=$ third order actuator dynamics

\section{Introduction}

A challenge in the design of adaptive control laws is addressing system stability and acceptable command following in the presence of uncertain, unmodeled actuator dynamics. In particular, degraded actuator performance can lead to poor tracking and even instability [1]. In real world applications, actuators seldom follow a first order model and their dynamics cannot be safely neglected as they can limit achievable stability of model reference adaptive control (MRAC). Therefore, it remains imperative that there exists a way to ensure safe interaction between the higher order actuator and the MRAC in the design phase.

Current literature attempts to address this by using MRAC control modifications. See [2, 3] where the authors consider a pseudo control hedging modification to include actuator dynamics in the design phase of MRAC. The authors of [4] 5] revisit pseudo-control hedging with a novel Lyapunov linear matrix inequality (LMI) method to ensure ideal bounded reference trajectories for a range of admissible bandwidths, natural frequency, and damping inherent in the actuator dynamics.

As discussed mainly in [5], the relationship between the damping and natural frequency can be bilinear. In other words, any variation in the damping can adversely affect the range of admissible natural frequencies required to maintain

${ }^{*}$ D. Wagner, is a researcher with the Faculty of Electrical Engineering, Czech Technical University in Prague, Technická 2, CZ-16626 Prague, Czech Republic, AIAA Student Member \{wagneda1@fel.cvut.cz\}

${ }^{\dagger}$ D. Henrion is a Professor with Faculty of Electrical Engineering, Czech Technical University in Prague, Technická 2, CZ-16626 Prague, Czech Republic and CNRS, LAAS, 7 avenue du colonel Roche, F-31400 Toulouse, France \{henrion@laas .fr

${ }^{\ddagger}$ M., Hromčík, is a Professor with the Faculty of Electrical Engineering, Czech Technical University in Prague, Technická 2, CZ-16626 Prague, Czech Republic \{hromcik@fel.cvut.cz\} 
closed-loop stability for higher order actuators and vice-versa. There is also a relationship between the MRAC learning rate and the closed-loop stability. Consequently, traditional Monte-Carlo methods can become costly for systems with these parameter uncertainties inherent in their dynamics. Every combination of these parameters must be simulated, and there remains risk of state-space explosion or unexplored, unsafe trajectories for medium and large scale systems.

Moment sum of squares (SOS) is a state-of-the-art method for verification and validation (VV) in aerospace. See [6, 7] where the authors focus on polynomial dynamical models and polynomial SOS Lyapunov candidate function. Occupation measures and LMI relaxations (called the moment sums of squares or Lasserre hierarchy) have also been used in VV of aircraft models with MRAC [8]. See also more recent development in using this framework where the authors [9, 10] exploit sparsity for ODEs using parsimony. Specifically, scaling issues brought about by optimizing directly over medium to large scale systems can be addressed by partitioning the dynamics.

The procedure remains similar to [10-[12]. First, our control law validation problem can be written as a polynomial dynamical optimization problem similar to that in optimal control. This problem is then written as its equivalent infinite dimensional linear programming (LP) problem by introducing occupation measures supported over admissible trajectories. Lastly, we relax the infinite dimensional LP problem of measures to a finite dimensional moment LMIs of truncated sequences.

The main contributions are the following:

- We validate an F-16 longitudinal model with MRAC and uncertain third order elevator dynamics with deflection saturation. The elevator contains bounded uncertainties inherent in its damping and natural frequency. Since uncertain parameters have an explicit representation in the space of occupation measures, we can identify numerically the worst case behavior caused by inherent uncertainties in damping and natural frequencies coming from the actuator. To avoid scaling problems, we exploit sparsity for ODEs to partition out the dynamics into a class of smaller systems that serve as control inputs for each other. In total, we are able to validate medium and large scale systems (up to 11 states) using this approach.

- Two examples are considered where the MRAC tolerates uncertainty in the natural frequency and damping, and one example where it obviously fails. Then, we compare our main results to those achieved using Monte-Carlo simulations and a search algorithm. The search algorithm locates the worst case damping and natural frequency behavior from the actuator.

Unlike the results of [2, 4, 5], we do not use the the pseudo control hedging modification to address actuator bandwidth uncertainty. Furthermore, none of these results take into account deflection saturation of the actuator. The results in [6, 7] provide only ultimate stability for aircraft models with linear feedback. Our previous work [8, 9] do not take into account uncertainties with a polynomial structure. In other words, our framework guarantees finite-time convergence for non-autonomous systems with piecewise dynamics. This is solution better than the solution provided by Barbalat's lemma [13] provided by the readily available Lyapunov certificate.

The organization of this work is as follows: Section III contains some necessary mathematical preliminaries, section IV discusses the short period of the F-16 with third order actuator dynamics, section $\mathrm{V}$ discusses our main results, and section $\mathrm{VI}$ gives our main conclusions and suggestions for future research.

\section{Mathematical Preliminaries}

We begin by very briefly stating some standard notation used for occupations measures. If $X$ is a compact subset of $\mathbb{R}^{n}, \mathscr{C}(X)$ denotes the space of continuous functions on $X$ and $\mathscr{M}(X)$ (resp., $\mathscr{M}_{+}(X)$ ) denotes the cone of (resp., non-negative) measures. We also assume that there is no relaxation gap $\left(J=J_{\infty}\right)$ between the polynomial dynamical optimization problem and its representation in the infinite dimensional space of measures. For the proceeding sections, we rely on the main theoretical results discussed extensively in [8, 9]. In particular, consider the polynomial dynamical optimization with piecewise polynomial different constraints

$$
\begin{array}{ll}
J=\inf _{h_{T}, h} & h_{T}(T, \mathbf{x}(t))+\int_{0}^{T} h(t, \mathbf{x}(t)) d t \\
\text { s.t. } & \dot{\mathbf{x}}(t)=\mathbf{f}_{j}(t, \mathbf{x}(t)), \quad \mathbf{x}(t) \in X_{j}, \quad j=1, \ldots, N \\
& \mathbf{x}(0) \in X_{0}, \quad \mathbf{x}(t) \in X_{T}, \quad t \in[0, T],
\end{array}
$$

with given costs $h, h_{T} \in \mathbb{R}[t, x]$, polynomial dynamics $f_{j} \in \mathbb{R}[t, x], j=1, \ldots, N$, state trajectory $\mathbf{x}(t)$ constrained in compact basic semialgebraic sets $X_{j}$, and initial (resp. terminal) given compact basic semialgebraic sets $X_{0}$ (resp., $X_{T}$ ). We also assume that the state space partitioning sets, or cells $X_{j}$, are such that all of their respective intersections have zero Lebesgue measure and all belong to a given compact semialgebraic set $X$. 
The nonconvex optimization problem eq. (1) can be approximated as a generic convex infinite dimensional LP problem of measures using parsimony

$$
\begin{aligned}
& J_{\infty}=\inf \int h_{T}(T, \mathbf{x}(t)) d \mu_{T}+\int h(t, \mathbf{x}(t)) d \mu \\
& \text { s.t. } \quad\left(\frac{\partial \mu}{\partial t}+\operatorname{divf}_{j} \mu_{j}\right)+\mu_{T}=\mu_{0} \\
& \left(\frac{\partial v}{\partial t}+\operatorname{divf}_{k} v_{k}\right)+v_{T}=v_{0} \\
& \sum_{j=1}^{N} \pi_{t, \mathbf{y} \# \mu_{j}}=\sum_{k=1}^{M} \pi_{t, \mathbf{y} \# v_{k}} \\
& \int \mu_{0}=1, \quad \int v_{0}=1,
\end{aligned}
$$

where div is the divergence operator and the infimum is with respect to the occupation measure $\mu, v \in \mathscr{M}_{+}([0, T] \times X)$, initial measure $\mu_{0}, v_{0} \in \mathscr{M}_{+}\left(\{0\} \times X_{0}\right)$, terminal measure $\mu_{T}, v_{T} \in \mathscr{M}_{+}\left(\{T\} \times X_{T}\right)$, terminal time $T>0$. Each measure $\mu_{j}$ (resp. $v_{k}$ ) supported on their respective cell $X_{j}$ (resp., $V_{k}$ ) so that the global occupation measure becomes

$$
\mu=\sum_{j=1}^{N} \mu_{j}, \quad v=\sum_{k=1}^{M} v_{k},
$$

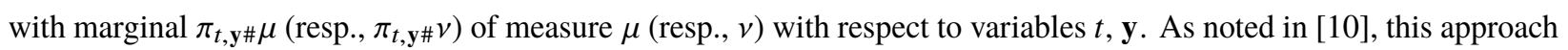
allows some flexibility with the computational limit caused by the largest moment SDP block. With appropriate partitioning strategies, we can solve problems with medium to large number of states.

\section{F-16 Short Period with Actuator Dynamics}

The short-period mode of an F-16 aircraft can be expressed by the dynamics

$$
\dot{\mathbf{x}}_{\mathbf{p}}(t)=A \mathbf{x}_{\mathbf{p}}(t)+B v(t), \quad \mathbf{x}_{\mathbf{p}}(0)=\mathbf{x}_{\mathbf{p} 0}
$$

where $\mathbf{x}_{\mathbf{p}}(t)=[\alpha(t) q(t)]$ are the short period dynamics, $\alpha(t) \in \mathbb{R}$ is the angle of attack, $q(t) \in \mathbb{R}$ is the pitchrate,

$$
A=\left[\begin{array}{ll}
-1.0189 & +0.9051 \\
+0.8223 & -1.0774
\end{array}\right], \quad B=\left[\begin{array}{l}
-0.0022 \\
-0.1756
\end{array}\right]
$$

are the open loop short period dynamics. Elevator actuator output $v(t) \in \mathbb{R}$ is described the third order unmodeled actuator dynamics

$$
\begin{aligned}
\dot{\mathbf{x}}_{\mathbf{c}}(t) & =\left(F+L\left(k_{1}, k_{2}\right)\right) \mathbf{x}_{\mathbf{c}}(t)+G u(t) \\
v(t) & =H \mathbf{x}_{\mathbf{c}}(t), \quad v(0)=v_{0}
\end{aligned}
$$

where $u(t) \in \mathbb{R}$ is a measurable control input,

$$
F=\left[\begin{array}{ccc}
0 & 1 & 0 \\
0 & 0 & 1 \\
-a \omega_{n}^{2} & \omega_{n}^{2}+2 \zeta \omega_{n} a ; & 2 \zeta \omega_{n}+a
\end{array}\right], \quad G=\left[\begin{array}{l}
0 \\
0 \\
1
\end{array}\right], \quad H=\left[\begin{array}{c}
a \omega_{n}^{2} \\
0 \\
0
\end{array}\right],
$$

$\zeta, \omega_{n}, a \in \mathbb{R}_{+}$are the damping, natural frequency, and gain respectively,. Matrix

$$
L=\left[\begin{array}{ccc}
0 & 1 & 0 \\
0 & 0 & 1 \\
-a k_{1}^{2} & k_{1}^{2}+2 k_{2} k_{1} a ; & 2 k_{2} k_{1}+a
\end{array}\right]
$$

contains inherent uncertainties within damping and natural frequency of the actuator dynamics described by bounded parameters $k_{1}, k_{2} \in\left[-k_{\max } k_{\max }\right], k_{\max } \in \mathbb{R}_{+}$. We assume that resulting matrix $(F+L)$ is Hurwitz, the DC gain between the control input $u(t)$ and actuator output $v(t)$ is unity, and pair $(A, B)$ is controllable. 


\section{A. Model Reference Adaptive Control}

Given a measurable command signal $c(t)$, we want to design a control law

$$
u(t)=u_{\mathrm{n}}(t)+u_{\mathrm{a}}(t)
$$

such that the combined nominal $u_{\mathrm{n}}(t)$ and adaptive control $u_{\mathrm{a}}(t)$ laws allow eq. (3) to asymptotically track reference model

$$
\dot{\mathbf{x}}_{\mathbf{r}}(t)=A_{\mathrm{r}} \mathbf{x}_{\mathbf{r}}(t)+B_{\mathrm{r}} c(t), \quad \mathbf{x}_{\mathbf{r}}(0)=\mathbf{x}_{\mathbf{r} 0}
$$

where $\mathbf{x}_{\mathbf{r}}(t)=\left[\alpha_{\mathrm{r}} q_{\mathrm{r}}\right]$ and the error dynamics

$$
\mathbf{e}(t)=\mathbf{x}_{\mathbf{p}}(t)-\mathbf{x}_{\mathbf{r}}(t)
$$

satisfies $\lim _{t \rightarrow \infty} \mathbf{e}(t)=0$. Matrices $A_{\mathrm{r}}=\left(A-B K_{1}\right)$ and $B_{\mathrm{r}}=B K_{2}$ are from the nominal controller

$$
u(t)=K_{1} \mathbf{x}_{\mathbf{p}}(t)+K_{2} c(t)
$$

with feedback/feedforward gains

$$
K_{1}=[+4.7432-2.3163], \quad K_{2}=[-4.3396]
$$

obtained using the LQR method [14, 15]. We assume that there exists a feedback matrix $K_{1}$ such that matrix $A_{\mathrm{r}}$ is Hurwtiz.

The adaptive controller

$$
u_{\mathrm{a}}(t)=-\hat{\mathbf{W}}^{T}(t) \Phi\left(\mathbf{x}_{\mathbf{p}}(t)\right),
$$

with the given basis function $\Phi_{i}\left(\mathbf{x}_{\mathbf{p}}(t)\right)=\left(1+e^{\mathbf{x}_{\mathbf{p} i}}\right)^{-1}, i=1,2$ and weight estimate $\hat{\mathbf{W}}(t)$, satisfies the update law

$$
\dot{\hat{\mathbf{W}}}(t)=\Gamma \Phi\left(\mathbf{x}_{\mathbf{p}}(t)\right) \mathbf{e}^{T}(t) P B_{\mathrm{p}}, \quad \hat{\mathbf{W}}(0)=\hat{\mathbf{W}}_{0}
$$

where $\Gamma=\operatorname{diag}([\epsilon 10]), \epsilon<<1$ is the learning rate and positive definite matrix $P$ is generated by the Lyapunov equality

$$
0=A_{\mathrm{r}}^{T} P+P A_{\mathrm{r}}+I_{2 \times 2} .
$$

Theorems that highlight the boundedness and long range stability of MRAC are discussed extensively in [13, 16]. For some discussion on control modifications, refer to [17]. For papers that discuss using these control modifications, refer to [8, 9].

\section{Main Results}

We wish to validate our existing closed loop polynomial aircraft model eq. (3) with actuator dynamics eq. (5) by finding the initial state that maximizes of the norm of the concave cost function $J=-\|\hat{\mathbf{e}}(T)\|_{2}^{2}$ where

$$
\hat{\mathbf{e}}(t)=\left[\begin{array}{c}
\alpha(t)-\alpha_{\mathrm{r}}(t) \\
q(t)-q_{\mathrm{ss}}(t)
\end{array}\right] \approx \mathbf{e}(t)
$$

approximates the error dynamics and $q_{s s}=\lim _{t \rightarrow \infty} q_{\mathrm{r}}(t)$. We also have given terminal time $T=25 \mathrm{~s}, c(t)=0.1$ and actuator constants

$$
a=5, \quad \omega_{n}=20, \quad \zeta=0.1,
$$

which are all guaranteed feasible solutions from [5]. If we can show that for every chosen initial state

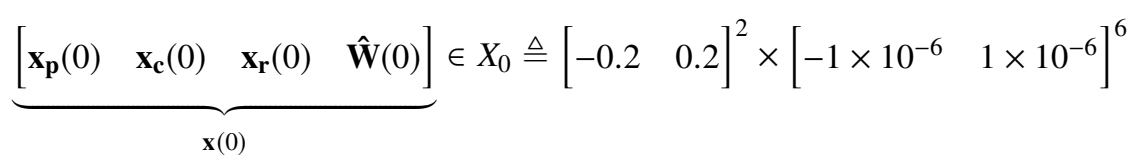


that all trajectories remain bounded in the box

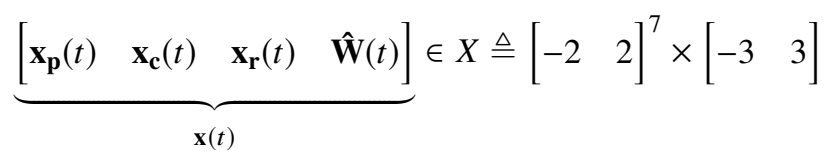

until they reach the terminal cost target $\hat{\mathbf{e}}(T) \in\left\{J \leq 4 \times 10^{-3}\right\}$, then our control law is validated. For our main results, we consider a $1 \%$ and $10 \%$ increase in the uncertainty the elevator dynamics by increasing $k_{\text {max }}$. We also include an example where the MRAC fails due to severe degraded actuator performance invoked by a $5000 \%$ increase in the dynamic uncertainty.

To include saturation in the elevator deflection, we partition the dynamics using locally affine functions in three cells $X_{j}, j=1, \ldots, 3$ corresponding to linear, upper saturation, lower regimes

$$
\begin{array}{ll}
X_{1} \triangleq\left\{\mathbf{x}(t) \in \mathbb{R}^{8}:|v(t)| \leq \theta_{\text {max }}\right\}, & v(t)=H \mathbf{x}_{\mathbf{c}}(t) \\
X_{2} \triangleq\left\{\mathbf{x}(t) \in \mathbb{R}^{8}: v(t) \geq \theta_{\text {max }}\right\}, & v(t)=\theta_{\text {max }} \\
X_{3} \triangleq\left\{\mathbf{x}(t) \in \mathbb{R}^{8}: v(t) \leq-\theta_{\text {max }}\right\}, & v(t)=-\theta_{\text {max }}
\end{array}
$$

where $\theta_{\text {max }}=\frac{25 \pi}{180}$.

With the combined short period dynamics eq. (3), actuator dynamics, in-state actuator uncertainties $k_{1}$ and $k_{2}$, and controller dynamics all partitioned into the saturation regimes, we can write eq. (1) as

$$
\begin{aligned}
& J=\inf _{\hat{\mathbf{e}}(T)}-\|\hat{\mathbf{e}}(T)\|_{2}^{2} \\
& \text { s.t. } \quad \dot{\mathbf{x}}_{\mathbf{p}}(t)=\mathbf{f}_{\mathbf{p}}\left(t, \mathbf{x}_{\mathbf{p}}(t), v(t)\right) \\
& \dot{\mathbf{x}}_{\mathbf{r}}(t)=\mathbf{f}_{\mathbf{r}}\left(t, \mathbf{x}_{\mathbf{r}}(t)\right) \text {, } \\
& {\left[\begin{array}{c}
\dot{\mathbf{x}}_{\mathbf{c}}(t) \\
\dot{\hat{\mathbf{W}}}(t)
\end{array}\right]=\mathbf{f}_{j}\left(t, \mathbf{x}_{\mathbf{c}}(t), \mathbf{x}_{\mathbf{p}}(t), \hat{\mathbf{W}}(t), \alpha_{\mathrm{r}}, k_{1}, k_{2}\right),} \\
& \mathbf{x}(t) \in X_{j}, \quad k_{1}, k_{2} \in\left[-k_{\text {max }} \quad k_{\text {max }}\right], \quad j=1, \ldots, 3 \\
& \mathbf{x}(0) \in X_{0}, \quad \mathbf{x}(t) \in X_{T}, \quad t \in[0, T],
\end{aligned}
$$

and the measure LP of parsimony eq. 2, as

$$
\begin{array}{ll}
J_{\infty}=\inf _{\mu_{T}} \quad-\int\|\hat{\mathbf{e}}(T)\|_{2}^{2} d \mu_{T} \\
\text { s.t. } \quad \frac{\partial \mu}{\partial t}+\operatorname{divf}_{\mathbf{p}} \mu\left(t, \mathbf{x}_{\mathbf{p}}, v\right)+\mu_{T}=\mu_{0} \\
& \frac{\partial v}{\partial t}+\operatorname{div} \mathbf{f}_{\mathbf{r}} v\left(t, \mathbf{x}_{\mathbf{r}}\right)+v_{T}=v_{0} \\
& \frac{\partial \xi}{\partial t}+\operatorname{divf}_{j} \xi\left(t, \mathbf{x}_{\mathbf{c}}, \hat{\mathbf{W}}, \mathbf{x}_{\mathbf{p}}, \alpha_{\mathrm{r}}, k_{1}, k_{2}\right)+\xi_{T}=\xi_{0} \\
& \pi_{t, \mathbf{x}_{\mathbf{p}}, v \# \mu}=\pi_{t, \mathbf{x}_{\mathbf{p}}, v \#} v \\
& \pi_{t, \mathbf{x}_{\mathbf{r}} \# v}=\sum_{j=1}^{3} \pi_{t, \mathbf{x}_{\mathbf{r}} \# \xi} \\
& \int \mu_{0}=1, \quad \int v_{0}=1, \quad \int \xi_{0}=1
\end{array}
$$

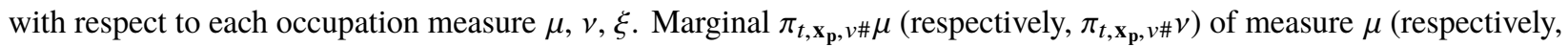
$v$ ) with respect to variables $t, \mathbf{x}_{\mathbf{p}}$, and $v$. Marginal $\pi_{t, \mathbf{x}_{\mathbf{r}} \#} \mu$ (respectively, $\pi_{t, \mathbf{x}_{\mathbf{r}} \# \xi} \xi$ of measure $\mu$ (respectively, $\xi$ ) with respect to variables $t, \mathbf{x}_{\mathbf{r}}$. As discussed in section III] we use this strategy to address scaling issues inherited from the problem. With this approach, we can reduce the overall size of the problem by 3 variables. As discussed in [8, 9], we also employ some basic scaling strategies so that all dynamics are normalized on the interval $\left[\begin{array}{ll}-1 & 1\end{array}\right]$. 

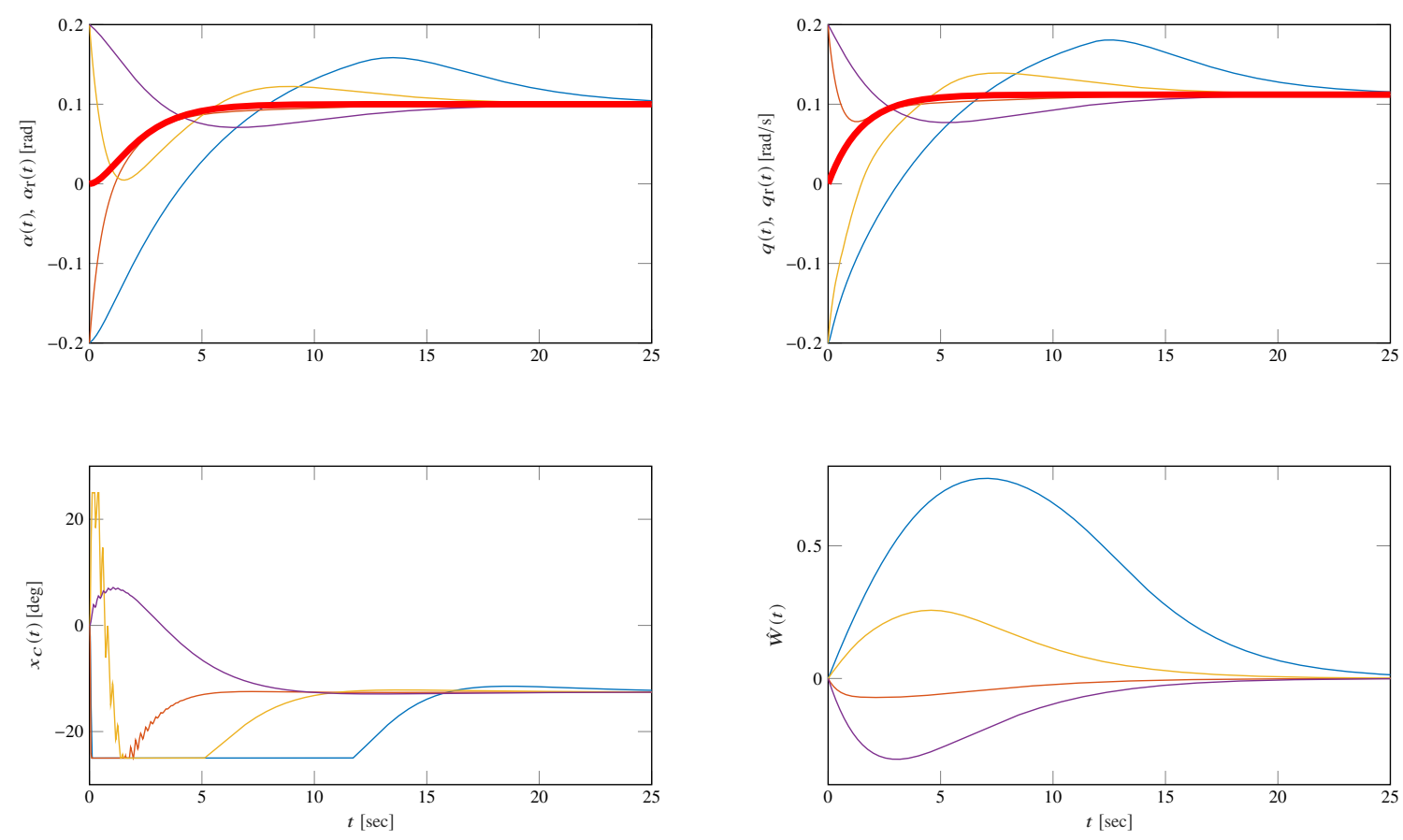

Fig. 1 F-16 Monte-Carlo $\left(k_{\max }=0.01, k_{1}=-0.01, k_{2}=0.01\right)$

\begin{tabular}{|c|c|c|c|c|c|c|}
\hline \multirow[b]{2}{*}{ Rel Ord } & \multicolumn{2}{|c|}{$k_{\max }=0.01$} & \multicolumn{2}{|c|}{$k_{\max }=0.1$} & \multicolumn{2}{|c|}{$k_{\max }=50$} \\
\hline & Upper Bnd $J$ & CPU [s] & Upper Bnd $J$ & CPU [s] & Upper Bnd $J$ & $\mathrm{CPU}[\mathrm{s}]$ \\
\hline 1 & $3.00 \times 10^{-2}$ & 2.81 & $3.10 \times 10^{-2}$ & 3.59 & 1.13 & 3.82 \\
\hline 2 & $9.78 \times 10^{-3}$ & $3.17 \times 10^{2}$ & $9.66 \times 10^{-3}$ & $3.36 \times 10^{2}$ & $1.03 \times 10^{-1}$ & $4.34 \times 10^{2}$ \\
\hline 3 & $1.98 \times 10^{-3}$ & $1.38 \times 10^{5}$ & $2.91 \times 10^{-3}$ & $1.15 \times 10^{5}$ & $2.86 \times 10^{-2}$ & $1.20 \times 10^{5}$ \\
\hline
\end{tabular}

Table 1 Gloptipoly 3 + MOSEK Upper Bounds for section $V$

The Monte-Carlo use a fixed time step and evenly distributed initial conditions to simulate the trajectories. For every simulation, we also use a search algorithm to find the matrix $L\left(k_{1}, k_{2}\right)$ that maximizes the terminal cost $J$. The worst case Monte-Carlo simulations and upper bounds are given in Figs. 1 to 3 and Table 2 , respectively,. Worst case parameters for natural frequency and damping are also shown in the captions.

As shown in Table 1, the upper bounds obtained using our framework (Gloptipoly $3+$ MOSEK) show the MRAC can tolerate a $0.1 \%$ and $10 \%$ uncertainty in damping and natural frequency. At $k_{\text {max }}=50$, the actuator performance degrades significantly and tracking is lost. We can also extract the time spent in the saturation regime, by extracting the mass of each occupation measure [11].

\section{Conclusion}

We validated a short period F-16 model with MRAC and a higher order elevator dynamics. The elevator contained parameter uncertainties and deflection saturation. We compared the results using our framework with those obtained using Monte-Carlo and a search algorithm. We also showed an example where the MRAC obviously fails due to poor

\begin{tabular}{rcc}
\hline$k_{\max }$ & $\begin{array}{c}\text { Upper Bound } \\
\boldsymbol{J} \text { (LQR + MRAC) }\end{array}$ & $\begin{array}{c}\text { CPU } \\
\text { Time [s] }\end{array}$ \\
\hline 0.01 & $3.13 \times 10^{-5}$ & $7.74 \times 10^{1}$ \\
0.1 & $3.13 \times 10^{-5}$ & $7.96 \times 10^{1}$ \\
50 & $2.27 \times 10^{-2}$ & $7.43 \times 10^{1}$ \\
\hline
\end{tabular}

Table 2 Monte-Carlo Upper Bounds for section $\mathbf{V}$ 

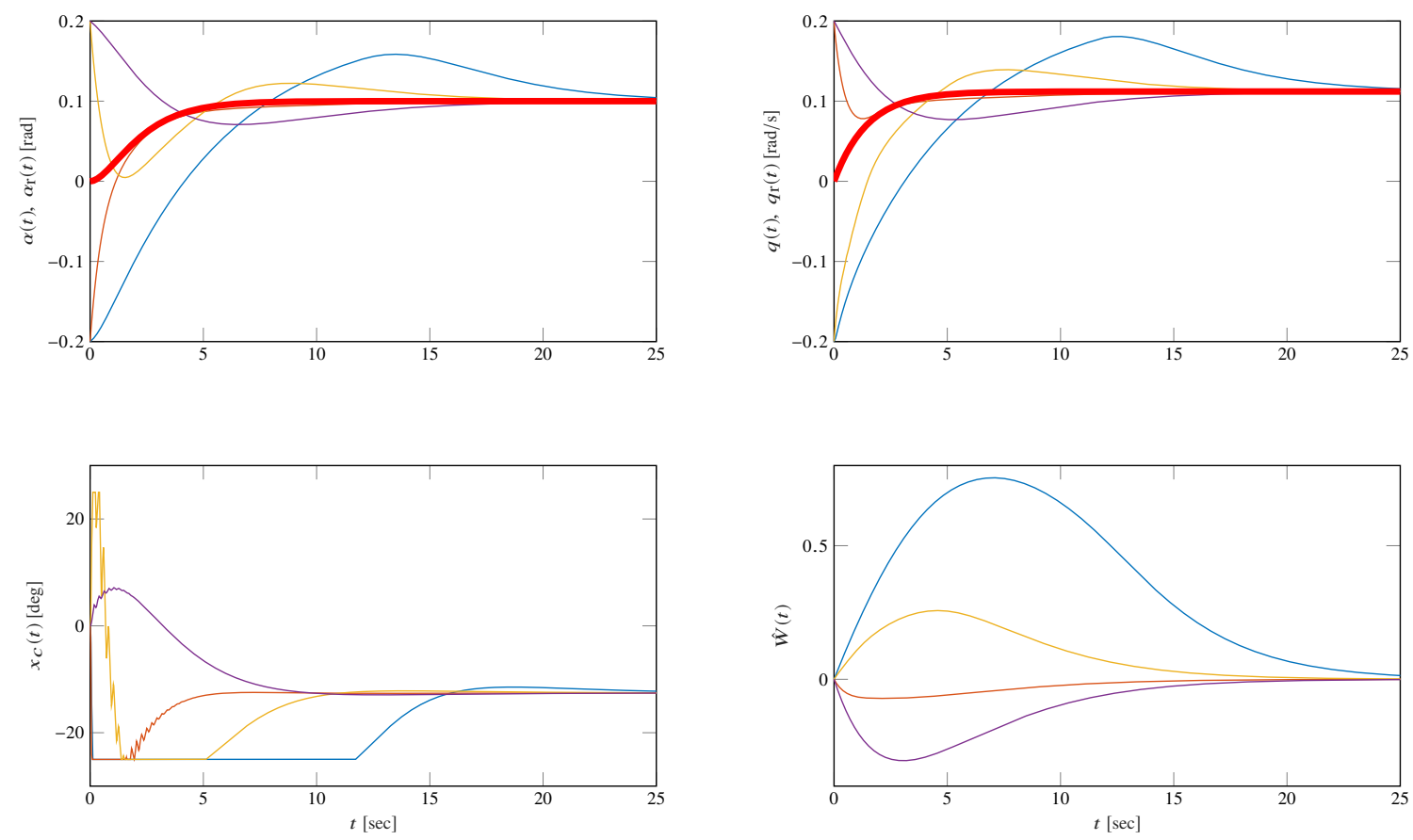

Fig. 2 F-16 Monte-Carlo $\left(k_{\max }=0.1, k_{1}=-0.1, k_{2}=0.1\right)$

performance from the elevator. For future results, we wish to find new ways to partition the dynamical problem to solve even larger scale systems and use region of attraction framework to find the numerical upper bounds for the uncertain parameters.

\section{References}

[1] Drydek, Z., Annaswamy, A., and Lavretsky, E., "Adaptive Control and the NASA X-15-3 Flight Revisited," IEEE Control Systems Magazine, Vol. 30, No. 3, 2010, pp. 32-48.

[2] Johnson, E. N., and Calise, A. J., "Pseudo-Control Hedging : a New Method for Adaptive Control," Redstone, AL, November 1-2 2000.

[3] Johnson, E. N., and Calise, A. J., "Limited authority adaptive flight control for reusable launch vehicles," AIAA Journal of Guidance, Control, and Dynamics, Vol. 26, No. 6, 2003, pp. 906-913.

[4] Gruenwald, B. C., Wagner, D., Yucelen, T., and Muse, J. A., "An LMI-Based Hedging Approach to Model Reference Adaptive Control with Actuator Dynamics," Columbus, OH, October 28-30 2015.

[5] Gruenwald, B., Yucelen, T., Muse, J. A., and Wagner, D., "Computing Stability Limits for Adaptive Control Laws with High-Order Actuator Dynamics," Automatica, Vol. 101, 2019, pp. 409-416.

[6] Chakraborty, A., "Nonlinear Robustness Analysis Tools for Flight Control Law Validation and Verification,” Ph.D. thesis, University of Minnesota, 2012.

[7] Chakraborty, A., Seiler, P., and Balas, G. J., "Susceptibility of F/A-18 Flight Controllers to the Falling-Leaf Mode: Nonlinear Analysis," AIAA Journal of Guidance, Control, and Dynamics, Vol. 32, No. 1, 2011, pp. 73-85.

[8] Wagner, D., Henrion, D., and Hromč ík, M., "Measures and LMIs for Adaptive Control Validation,” Nice FR, December 11-13 2019.

[9] Wagner, D., Henrion, D., and Hromčík, M., “Measures and LMIs for Lateral F-16 MRAC Validation,” Denver, CO, July 1-3 2020.

[10] Tacchi, M., Cardozo, C., Henrion, D., and Lasserre, J. B., “Approximating regions of attraction of a sparse polynomial differential system,” Berlin, DE, July 12-17 2020. 

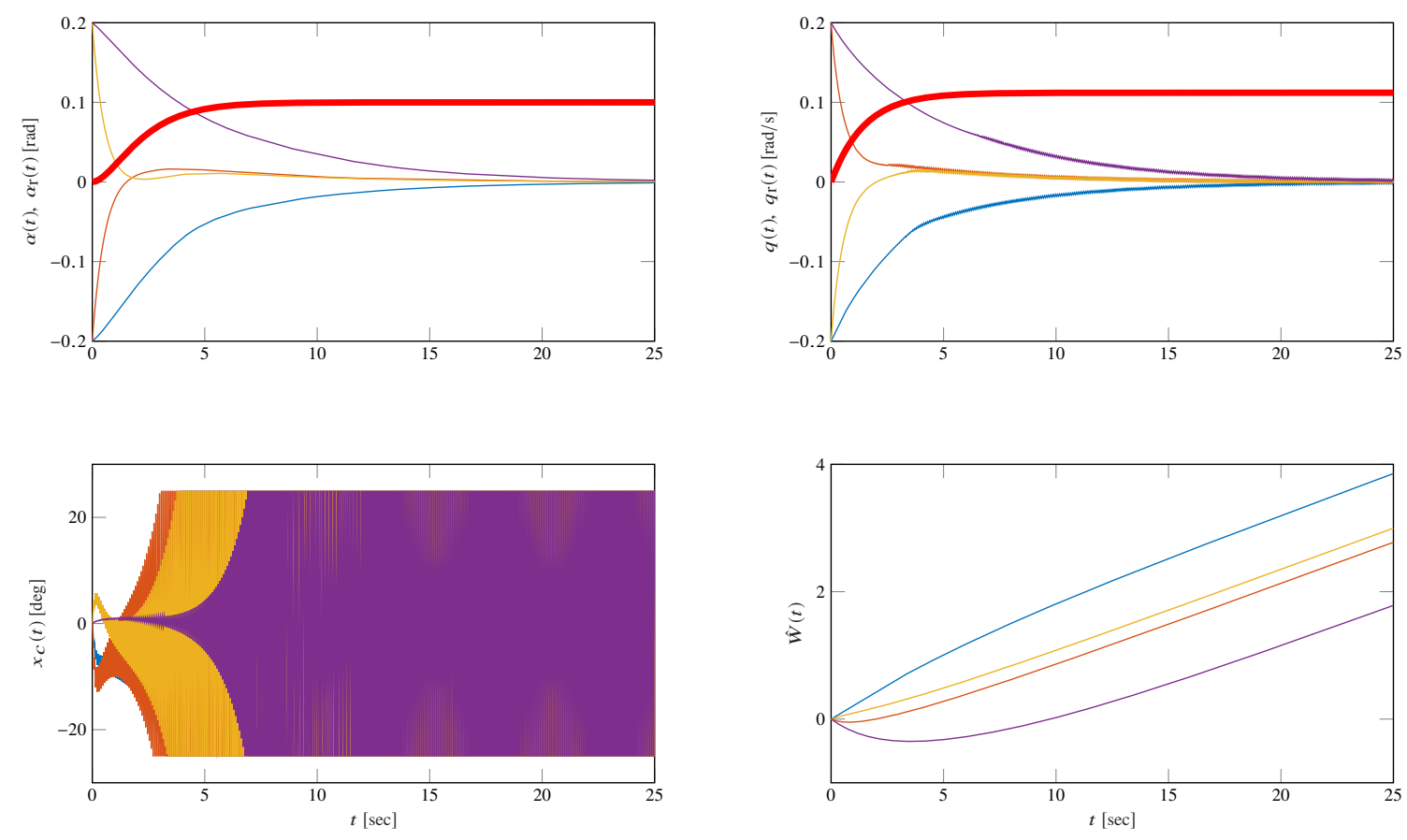

Fig. 3 F-16 Monte-Carlo $\left(k_{\max }=50, k_{1}=-50, k_{2}=0\right)$

[11] Henrion, D., Ganet-Schoeller, M., and Bennani, S., "Measures and LMI for space launcher robust control validation," Copenhagen, DK, 2011, pp. 236-241. https://doi.org/10.3182/20120620-3-DK-2025.00016.

[12] "Optimization on linear matrix inequalities for polynomial systems control," Les cours du CIRM, Vol. 3, No. 1, 2013 , pp. 1-44.

[13] Haddad, W. M., and Chellaboina, V. S., Nonlinear dynamical systems and control: A Lyapunov-based approach, Princeton University Press, Princeton, NJ, 2011. https://doi.org/10.2307/j.ctvcm4hws

[14] Fravolini, M. L., Arabi, E., and Yucelen, T., "A Model Reference Adaptive Control Approach for Uncertain Dynamical Systems with Strict Component-wise Performance Guarantees,” Kissimmee, FL, January 8-12, 2018.

[15] Stevens, B. L., Lewis, F. L., and Johnson, E. N., Aircraft control and simulation: Dynamics, controls design, and autonomous systems: Third edition, $3^{\text {rd }}$ ed., Wiley, NY, 2015. https://doi.org/10.1002/9781119174882

[16] Lavretsky, E., and Wise, K., Robust and Adaptive Control With Aerospace Applications, Springer-Verlag, London, 2013.

[17] Narendra, K. S., and Annaswamy, A. M., "A New Adaptive Law for Robust Adaptation Without Persistent Excitation," IEEE Transactions on Automatic Control, Vol. 32, No. 2, 1987, pp. 134-145. https://doi.org/10.1109/TAC.1987.1104543

[18] Noel, J., Renson, L., Kerschen, G., Peeters, B., Manzato, S., and Debille, J., "Nonlinear Dynamic Analysis of an F-16 Aircraft Using GVT Data,” Bristol, UK, 2014.

[19] Tallant, G. S., Bose, P., Buffington, J. M., Crum, V. W., Hull, R. A., Johnson, T., Krogh, B., and Prasanth, R., "Validation \& Verification of Intelligent and Adaptive Control Systems,” Big Sky, MT, March 5-12 2005.

[20] Heller, M., Niewoehner, P. K., and Lawson, P. K., "On the validation of safety critical aircraft systems, part I: An overview of analytical \& simulation methods," Austin, TX, August 11-14 2003.

[21] Whitaker, H. P., Yamron, J., and Kezer, A., "Design of Model Reference Control Systems for Aircraft,” Tech. rep., Instrumentation Laboratory, Massachusetts institution of Technology, Cambridge, MA, 1958.

[22] Osburn, P. V., Whitaker, H. P., and Kezer, A., "New Developments in the Design of Adaptive Control Systems," Tech. rep., Institution of Aeronautical Sciences, 1961.

[23] Kokotović, P. V., Foundations of Adaptive Control, Springer-Verlag, New York, 1991. 
[24] Krstić, M., Kanellakopoulos, I., and Kokotović, P. V., Nonlinear and Adaptive Control Design, Wiley, New York, 1995.

[25] Tao, G., Adaptive Control Design and Analysis, John Wiley \& Sons, New York, 2003.

[26] Ioannou, P., and Fidan, B., Adaptive Control Tutorial, SIAM, Philadelphia, PA, 2006.

[27] Hovakimyan, N., and Cao, C., $\mathcal{L}_{1}$ Adaptive Control Theory: Guaranteed Robustness with Fast Adaptation, SIAM, Philadelphia, PA, 2010.

[28] Sastry, S., and Bodson, M., Adaptive Control: Stability, Convergence and Robustness, Dover Publications, New York, 2011.

[29] Narendra, K. S., and Annaswamy, A. M., Stable Adaptive Systems, Dover Publications, New York, 2012.

[30] Åström, K. J., and Wittenmark, B., Adaptive Control, Dover Publications, New York, 2013.

[31] Wise, K. A., Lavretsky, E., and Hovakimyan, N., "Adaptive Control of Flight: Theory, Applications, and Open Problems," Minneapolis, MN, June 14-16 2006.

[32] Anderson, B. D., and Dehghani, A., "Challenges of adaptive control-past, permanent and future," Annual Reviews in Control, Vol. 32, No. 2, 2008, pp. 123-135.

[33] Black, W. S., Haghi, P., and Ariyur, K. B., “Adaptive Systems: History, Techniques, Problems, and Perspectives,” Systems, Vol. 2, No. 4, 2014, pp. 606-660.

[34] Tao, G., "Multivariable adaptive control: A survey," Automatica, Vol. 50, No. 411, 2014, pp. 2737-2754.

[35] Bosworth, J., and Williams-Hayes, P., "Flight Test Results from the NF-15B IFCS Project with Adaptation to a Simulated Stabilator Failure,” Rohnert Park, CA, May 7-10 2007.

[36] Bosworth, J., and Williams-Hayes, P., "Flight Test Results from the NF-15B Intelligent Flight Control System (IFCS) Project with Adaptation to a Simulated Stabilator Failure," May 7-10 2007. https://doi.org/10.2514/6.2007-2818. URL https://arc.aiaa.org/doi/abs/10.2514/6.2007-2818

[37] Wall, J. H., Orr, J. S., and VanZweiten, T., "Space Launch System Implementation of Adaptive Augmenting Control," Breckenridge, CO, January 31-February 52014.

[38] Adbalmoaty, M., Henrion, D., and Rodrigues, L., "Measures and LMIs for optimal control of piecewise-affine systems," Zurich, CH, January 17-19 2013.

[39] Sturm, J., "Using SeDuMi 1.02, a MATLAB toolbox for optimization over symmetric cones," Optimization Methods and Software, Vol. 11-12, 1999, pp. 625-653.

[40] ApS, M., "The MOSEK optimization toolbox for Matlab manual,”, 2019. www.mosek.com/ (accessed September 18, 2020).

[41] Lavretsky, E., "Combined/composite model reference adaptive control," IEEE Transactions on Automatic Control, Vol. 54, No. 11, 2009, pp. 2692-2697. https://doi.org/10.1109/TAC.2009.2031580

[42] Calise, A. J., Yucelen, T., Muse, J. A., and Yang, B. J., “A loop recovery method for adaptive control,” Chicago, IL, 2009. https://doi.org/10.2514/6.2009-5967

[43] Jacklin, S. A., “Closing certification gaps in adaptive flight control software,” Honolulu, HI, August 18-21 2012.

[44] Nguyen, N., and A, J. S., Neural Net Adaptive Flight Control Stability, Verification and Validation Challenges, and Future Research, Springer-Verlag, Berlin, 2015.

[45] Calise, A. J., Lee, S., and Sharma, M., "Development of a reconfigurable flight control law for tailless aircraft," AIAA Journal of Guidance, Control, and Dynamics, Vol. 24, No. 5, 2011, pp. 896-902. https://doi.org/10.2514/2.4825

[46] Yucelen, T., and Calise, A. J., "Derivative-free model reference adaptive control of a generic transport model," Toronto, CA, 2010. https://doi.org/10.2514/6.2010-8402

[47] AIAA Intelligent Systems Technical Committee, "Roadmap For Intelligent Systems In Aerospace,” Tech. rep., AIAA, 2016.

[48] Gabernet, A. F., "Controllers for Systems with Bounded Actuators : Modeling and control of an F-16 aircraft," Ph.D. thesis, University of California, Irvine, 2007. 
[49] Sharma, M., Lavretsky, E., and Wise, K. A., "Application and flight testing of an adaptive autopilot on precision guided munitions," Keystone, CO, 2006. https://doi.org/10.2514/6.2006-6568

[50] Morelli, E. A., "Global nonlinear parametric modeling with application to F-16 aerodynamics,” Philadelphia, PA, 1998, pp. 997-1001. https://doi.org/10.1109/ACC.1998.703559

[51] Rohrs, C., Valavani, L., Athans, M., , and Stein, G., "Robustness of continuous-time adaptive control algorithms in the presence of unmodeled dynamics," IEEE Transactions on Automatic Control, Vol. 30, No. 9, 1985, pp. 881-889.

[52] Calise, A., Hovakimyan, N., and Idan, M., "Adaptive output feedback control of nonlinear systems using neural networks," Automatica, Vol. 38, No. 8, 2001, pp. 1201-1211.

[53] Hovakimyan, N., Nardi, F., Calise, A., and Kim, N., "Adaptive output feedback control of uncertain nonlinear systems using single-hidden-layer neural networks," IEEE Transactions on Neural Networks, Vol. 13, No. 6, 2002, pp. 1420-1431.

[54] Tong, S., Wang, T., Li, Y., and Zhang, H., "Adaptive output feedback control of uncertain nonlinear systems using single-hiddenlayer neural networks," IEEE Transactions on Neural Networks, Vol. 13, No. 6, 2014, pp. 1420-1431.

[55] Tong, S., Wang, T., and Li, Y., "Fuzzy adaptive actuator failure compensation control of uncertain stochastic nonlinear systems with unmodeled dynamics," IEEE Transactions on Fuzzy System, Vol. 22, No. 3, 2014, pp. 563-574.

[56] Li, Y., Sui, S., and Tong, S., "Adaptive fuzzy control design for stochastic nonlinear switched systems with arbitrary switchings and unmodeled dynamics," IEEE Transactions on Cybernetics, Vol. 47, No. 2, 2016, pp. 403-414.

[57] Matsutani, M., Annaswamy, A. M., Gibson, T. E., and Lavretsky, E., "Trustable autonomous systems using adaptive control," Orlando, FL, December 12-15 2011.

[58] Matsutani, M., and Annaswamy, A. M., "Guaranteed delay margins for adaptive control of scalar plants," Maui, HI, December 10-13 2012.

[59] Matsutani, M., Annaswamy, A. M., and Lavretsky, E., "Robust adaptive control in the presence of unmodeled dynamics: A counter to Rohrs's counterexample," Boston, MA, August 19-22 2013.

[60] Merve Dogan, K., Gruenwald, B. C., Yucelen, T., and Muse, J. A., "Relaxing the stability limit of adaptive control systems in the presence of unmodelled dynamics," International Journal of Control, Vol. 91, No. 8, 2018, pp. 1774-1784.

[61] Henrion, D., Lasserre, J. B., and Löfberg, J., "GloptiPoly 3: moments, optimization and semidefinite programming," Optimization Methods and Software, Vol. 24, No. 4-5, 2009, pp. 761-779.

[62] SeDuMi, "SeDuMi: A linear/quadratic/semidefinite solver for Matlab and Octave,", 2019. https://github.com/sqlp/sedumi. (accessed September 18, 2020).

[63] Lasserre, J. B., Moments, positive polynomials and their applications, Imperial College Press, London, 2009.

[64] Merve Dogan, K., Gruenwald, B. C., Yucelen, T., and Muse, J. A., "Relaxing the stability limit of adaptive control systems in the presence of unmodelled dynamics," International Journal of Robust and Nonlinear Control, Vol. 91, No. 8, 2016, pp. 1774-1784.

[65] Merve Dogan, K., Yucelen, T., and Muse, J. A., "Adaptive Control with Unmeasurable Coupled Dynamics: An Asymptotic Decoupling Approach,” Denver, CO, July 1-3 2020. Accepted for publication.

[66] Merve Dogan, K., Yucelen, T., Ristevski, S., and Muse, J. A., "Distributed Adaptive Control of Uncertain Multiagent Systems with Coupled Dynamics," Denver, CO, July 1-3 2020. Accepted for publication.

[67] Merve Dogan, K., Yucelen, T., and Muse, J. A., "Performance Guarantees in Adaptive Control of Uncertain Systems with Unmodeled Dynamics,” Orlando, FL, January 6-10 2020.

[68] Fravolini, M., Merve Dogan, K., and Yucelen, T., "Quantification of Tolerable Parametric and Dynamic Uncertainty for Robust Model Reference Adaptive Control Systems,” Orlando, FL, January 6-10 2020.

[69] Merve Dogan, K., and Yucelen, J. A., T Muse, “Adaptive Control of Dynamical Systems with Unstructured Uncertainty and Unmodeled Dynamics,” Philadelphia, PA, July 10-12 2019.

[70] Merve Dogan, K., Yucelen, T., Yildirim, J., and Muse, J. A., "Experimental Results of a Model Reference Adaptive Control Law on an Uncertain System with Unmodeled Dynamics," San Diego, CA, January 7-11 2019.

[71] Merve Dogan, K., Yucelen, T., Gruenwald, B. C., and Muse, J. A., "On Model Reference Adaptive Control for Uncertain Dynamical Systems with Unmodeled Dynamics,” Las Vegas, NV, December 12-14 2016. 